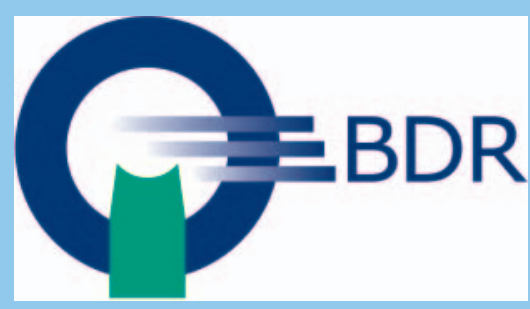

\title{
Kontrastmittel-Pauschalen in der (Medien)-Kritik
}

\section{Erklärung des BDR zu den Reportagen von Süddeutscher Zeitung, WDR und NDR zum Thema Kontrastmittel}

Die vom Recherche Netzwerk (SZ, WDR, NDR) ermittelten und veröffentlichten Darstellungen (Sendung Panorama vom 1.8.19) sind unvollständig und in ihrer Aussage unzutreffend.

Die Verträge über Kontrastmittelbezug sind immer, egal wie gestaltet, Verträge zwischen Kassenärztlichen Vereinigungen (KVen) und Krankenkassen (meist vertreten durch die AOK, aber auch Barmer und sonstige Krankenkassen). Radiologen sind als zugelassene oder angestellte Ärzte zwangsläufig Mitglieder der jeweiligen KV und damit an deren Vertragsgestaltung mit den Krankenkassen gebunden.

Bei Kontrastmitteln handelt es sich um verordnungspflichtige Arzneimittel, die aber von der Apothekenpflicht befreit sind. In der Vergangenheit wurden die Kontrastmittel auf dem Wege des „Sprechstundenbedarfs“ direkt vom Radiologen auf Vorrat beim Hersteller oder Händler auf vorgeschriebenen Wegen bestellt. Damit wurden die Apotheken als potentielle Lieferanten zur Kostensenkung umgangen. Die Preise wurden der „Lauer-Taxe“1 entnommen und von den Krankenkassen auch so bezahlt, Mengenrabatte waren nicht vorgesehen oder möglich. Der Radiologe füllte hierfür lediglich ein entsprechendes Rezept aus, gab dieses an die Kontrastmittelhersteller oder Händler weiter, die den Radiologen direkt belieferten. Es gab keinerlei Rabatte. Entsprechend kostete ein Liter Kernspin-Kontrastmittel etwa $7000 €$. Zur gleichen Zeit kostete das identische Kon-

1 Die Lauer-Taxe ist ein Nachschlagewerk für alle pharmazeutischen Berufsgruppen, Apotheken, Pharmaunternehmen und Großhändler, gesetzliche und private Krankenversicherungen. trastmittel im Krankenhauseinkauf im Paket mit anderen Medikamenten und zusätzlich im Wettbewerb (Verhandlungsweg zwischen Krankenhaus und Pharma-Industrie), deutlich weniger.

Die Krankenkassen haben deshalb versucht, die Preise im ambulanten Sektor durch Ausschreibungen zu reduzieren. Dies war zunächst aber wenig effektiv. Die Angebote der Ausschreibungsgewinner lagen meist nur geringfügig unter dem ursprünglichen Preis. Eine Transparenz kam zudem nicht zu Stande, da die erzielten Preise nach europäischem Ausschreibungsrecht nicht veröffentlicht werden dürfen. In der Folge versuchten die Krankenkassen dann ältere, inzwischen sogar teilweise obsolete (oder in der Anwendung stark eingeschränkte) Kontrastmittel in die Ausschreibungslose hineinzustellen oder wirkstoffübergreifende Ausschreibungslose zu bilden, um so bessere Preise zu erzielen.

Dagegen haben sich die Radiologen, zum Erhalt der Therapiefreiheit im Sinne der ihnen anvertrauten Patienten, gewehrt und verlangt, dass nur gleiche Substanzen, also gleiche Wirkstoffe, in einem Ausschreibungslos zusammengelegt werden dürften. Schließlich müsste sonst der Radiologe auch schlechtere Kontrastmittel anwenden, die er selbst gar nicht verordnen wollte und hierfür letztlich auch haften! Die Krankenkassen haben den Weg der wirkstoffübergreifenden Ausschreibung dennoch in mehreren Bundesländern (z. B. RLP, Saarland, SchleswigHolstein), auch mit Unterstüt-zung der jeweiligen Gesundheitsministerien, nicht verlassen und ihrerseits jegliche Haftung abgelehnt.

Abweichend wurde in Bayern schon vor 20 Jahren ein anderer Weg beschritten.
Der Radiologe sollte die Kontrastmittel direkt beziehen und bezahlen, um so den Wettbewerb zwischen Herstellern und/ oder Händlern zu generieren. Zum Ausgleich erhielt der Radiologe eine Abrechnungsziffer (Pauschale) für den Einkauf, die Bevorratung, die Sicherstellung der medizinischen Anwendung und die korrekte Indikationsstellung im Einzelfall, ausgehandelt zwischen den Krankenkassen und der jeweiligen KV. Das Preisrisiko ging damit auf die Ärzte über. Ein Weg übrigens, der auch in vielen anderen Fachgruppen, mit anderen Medizinprodukten praktiziert wird (z. B. bei der Dialyse, Einkauf von Linsen durch Augenärzte etc.). Mit dem Pauschalen-Modell entstand für die Hersteller und Händler von Kontrastmitteln zum ersten Mal eine Wettbewerbssituation und die Preise kamen in Bewegung. Schon die ersten Pauschalen lagen für die Krankenkassen deutlich unter den Preisen der Lauer-Taxe und bedeuteten erhebliche Einsparungen für die Krankenkassen. Diese konnten dann im weiteren Verlauf, mithilfe der Radiologen, noch weiter gesenkt werden. In Bayern wurden die Pauschalen zu einem „Teil der Vergütung“. Dieses Modell „Pauschale für Kontrastmittel“ wurde dann langsam verfeinert und auch von anderen Bundesländern (KVen) eingeführt. Zuletzt hat dann auch die AOK Rheinland in der KV Nordrhein diesen Weg beschritten und die bis dahin von den Radiologen bekämpfte wirkstoffübergreifende Ausschreibung verlassen, um hiermit eigene Haftungsrisiken auszuschalten.

Ergänzt wurde die Pauschalen-Regelung meist durch eine strikte Mengenbegrenzung, die eine Steigerung des Kontrastmittelverbrauches auch wirksam verhinderte. Heute können Bundesländer (KVen) wie 
Hamburg, Niedersachsen und auch Bremen, nachweisen, dass keinerlei Mehrverbrauch von Kontrastmitteln durch die Pauschalen entstanden ist. Jeder anderslautende Vorwurf, der Radiologen eigennützige Manipulationen unterstellt, ist unseriös und anhand von Abrechnungsdaten zu widerlegen. Der Anteil der Kontrastmittelpauschalen je Untersuchung ist z. B. in Hamburg seit Beginn der Regelung völlig konstant. Der Preis wurde mit Einführung der Pauschalen gegenüber der Lauer-Taxe (ursprünglicher Preis bei $7000 € / l)$ um $40 \%$ auf aktuell etwa $3900 € /$ pro Liter abgesenkt. Dadurch aber waren die Radiologen in der Verantwortung bei Herstellern oder Händlern Marktpreise zu erzielen. Egal welcher Preis geboten wurde, der Radiologe erhielt für die Untersuchung mit Kontrastmittel immer nur diese Pauschale. Je nach Praxisgröße, Einkaufsmenge und Patientenauswahl (die Untersuchungen benötigen verschiedene Kontrastmittel und Dosierungen), sind Gewinne oder Verluste für die Radiologen möglich.
Für die Krankenkassen und KVen bedeutet dieses Modell aber eine feste kalkulierbare Größe. Zudem wurden in den PauschalenVerträgen feste Laufzeiten zwischen Kassen und KV vereinbart, sie sehen selbstverständlich auch Anpassungsregelungen vor.

Entgegen der Darstellung in Panorama und Tagesschau ist somit nicht „Geld der Versicherten in Millionenhöhe verschwendet“ worden, sondern durch die Pauschalen-Modelle konnten viele Millionen Kassengelder eingespart werden. Das Pauschalen-Modell ist unzweifelhaft rechtskonform und sichert Patienten und Ärzten die Therapiefreiheit! Pauschalen sind in der Gebührenordnung für Ärzte (EBM) eine übliche Vergütungskomponente. Radiologen haben mit dem Pauschalen-Modell weder unzulässige Gewinne erzielt, noch die von Panorama genannten Preisspannen zur Gewinnsteigerung realisiert, da eine Pauschale eben pauschal mehrere Kostenfaktoren abzudecken hat. Radiologen haben sich das Kontrastmittel-PauschalenModell nicht gewünscht.

Soweit die ganze Geschichte des "Skandals“, erzählt von den Betroffenen. Sicher kaum reiBerisch genug, um Auflage zu schaffen, aber eben ein Bericht über alltägliche medizinische Versorgung in Deutschland.

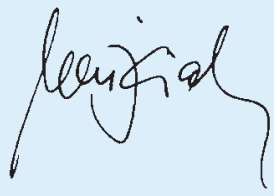

Dr. Detlef Wujciak

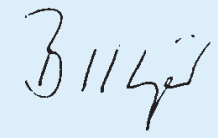

Dr. Andreas Bollkämper 